\title{
Crianças com câncer e suas famílias
}

\author{
CHILDREN WITH CANCER AND THEIR FAMILIES
}

\author{
NIÑOS CON CÁNCER Y SUS FAMILIAS
}

\section{Lucila Castanheira Nascimento ${ }^{1}$, Semiramis Melani Melo Rocha², Virginia Hellen Hayes ${ }^{3}$,Regina Aparecida Garcia de Lima ${ }^{4}$}

\section{RESUMO}

O objetivo deste estudo é revisar a literatura relativa à criança com câncer e sua família, a fim de identificar temas que têm sido pesquisados e levantar indicadores de necessidades, subsidiando a sistematização da assistência de enfermagem. A coleta de dados sistemática foi realizada em bancos de dados informatizados, entre $1997 \mathrm{e}$ 2002, utilizando-se as palavraschave child, cancer, chronic illness/ disease, family e nursing. Realizouse, também, busca não-sistemática de publicações científicas. Os resultados foram apresentados em três temas: impacto do câncer infantil no sistema familiar; processo de adaptação e estratégias de enfrentamento utilizadas pelos pais diante da doença e o processo de perda e luto diante da morte da criança. A revisão demonstrou que a enfermagem está construindo um conhecimento específico sobre as necessidades individuais, culturais e regionais das famílias de crianças com câncer, para uma assistência de enfermagem que considere o cuidado de acordo com a singularidade de cada caso.

\section{ABSTRACT}

This study is aimed at reviewing the existing literature about children with cancer and their families in order to identify themes that have been researched and to survey indicators of need, thus giving subsidies for the systematization of nursing care. Systemized data collection was carried out in computerized databases between 1997 and 2002 using the keywords child, cancer, chronic illness/disease, family and nursing. A non-systemized research of scientific publications was also carried out. Results are grouped in three themes: impact of the child's cancer on the family system; adaptation process and coping strategies used by the parents in the face of the illness; and the process of loss and mourning in view of the child's death. The review demonstrated that nursing is constructing specific knowledge about the individual, cultural and regional needs of families of children with cancer, with a view to a nursing assistance that considers care in accordance to the singularity of each case.

\section{KEY WORDS}

\section{DESCRITORES}

Enfermagem pediátrica.

Doença crônica.

Neoplasias.

Criança.

Família.

Enfermagem familiar.
Pediatric nursing.

Chronic disease.

Neoplasms.

Child.

Family.

Family nursing.

\section{RESUMEN}

El objetivo en este estudio fue revisar la literatura relativa al niño con cáncer y su familia, a fin de identificar temas que hayan sido investigados y buscar indicadores de necesidades, subsidiando la sistematización de la asistencia de enfermería. La recolección sistemática de datos fue realizada en bancos de datos informatizados, entre 1997 y 2002 , utilizándose las palabras clave child, cancer, chronic illness/ disease, family e nursing. Se realizó, también, búsqueda no-sistemática de publicaciones científicas. Los resultados fueron presentados en tres temas: impacto del cáncer infantil en el sistema familiar; proceso de adaptación y estrategias de enfrentamiento utilizadas por los padres frente a la enfermedad y el proceso de pérdida y duelo frente a la muerte del niño. La revisión demostró que la enfermería está construyendo un conocimiento específico sobre las necesidades individuales, culturales y regionales de las familias de niños con cáncer, para una asistencia de enfermería que considere la atención de acuerdo con la singularidad de cada caso.

\section{DESCRIPTORES}

Enfermería pediátrica.

Enfermedad crónica.

Neoplasmas.

Niño.

Familia.

Enfermería de la familia.
1 Enfermeira. Professor Doutor junto à Escola de Enfermagem de Ribeirão Preto da Universidade de São Paulo (EERP/USP). lucila@eerp.usp.br

2 Enfermeira, Professor Titular junto à EERP/USP.

$3 \mathrm{RN}, \mathrm{PhD}$, Professor, University of Victoria, B.C., Canada.

4 Enfermeira, Professor Associado junto à EERP/USP 
Lucila C. Nascimento

Semiramis M. M. Rocha

Virginia H. Hayes

Regina A. G. de Lima

\section{INTRODUÇÃO}

No grupo populacional de zero a dezoito anos incidem várias doenças crônicas com maior ou menor prevalência, de acordo com as especificidades de cada faixa etária e região geográfica. Dentre as doenças crônicas infantis, o câncer se destaca pela sua alta incidência e repercussões na vida da criança e sua família e, por isso, optamos por selecioná-lo como objeto de estudo deste trabalho.

Famílias de crianças com câncer, muitas vezes, sentem-se impotentes para satisfazerem as necessidades relacionadas aos cuidados de saúde de suas crianças e de sustentarem suas vidas familiares. Capacitar essas famílias é uma intervenção que pode ser feita pelos enfermeiros. Este estudo tem o objetivo de revisar a literatura nacional e internacional, relativa à criança com câncer e sua família, a fim de identificar temas que têm sido pesquisados e levantar indicadores de necessidades, subsidiando a sistematização da assistência de enfermagem.

\section{METODOLOGIA}

Trata-se de revisão da literatura, abrangendo o período de 1997 a 2002, nos seguintes bancos de dados informatizados: Cumulative Index of Nursing and Allied Health Literature (CINAHL), Literatura da América Latina e do Caribe em Ciências da Saúde (LILACS), base de dados da literatura internacional - USA (MEDLINE) e Health Star.

Na busca sistemática, utilizamos as palavraschave child, cancer, chronic illness/chronic disease, family e nursing. Após a captação, foi realizada nova busca com o cruzamento das palavras-chave, resultando em vinte e sete artigos que atendiam aos objetivos deste estudo. Realizamos, também, busca não-sistemática de publicações científicas referentes à temática que pudessem contribuir para a delimitação das necessidades de assistência à criança com câncer e sua família.

\section{RESULTADOSE DISCUSSÃO}

Para maior clareza e facilidade de leitura, apresentamos, a seguir, a revisão da literatura enfocando a criança com câncer e sua família, agrupada nos seguintes temas: impacto do câncer infantil no sistema familiar; processo de adaptação e estratégias de enfrentamento utilizadas pelos pais diante da doença e o processo de perda e luto frente à morte da criança.
Impacto do câncer infantil no sistema familiar:

A família e a criança enfrentam problemas como longos períodos de hospitalização, reinternações freqüentes, terapêutica agressiva com sérios efeitos indesejáveis advindos do próprio tratamento, dificuldades pela separação dos membros da família durante as internações, interrupção das atividades diárias, limitações na compreensão do diagnóstico, desajuste financeiro, angústia, dor, sofrimento e o medo constante da possibilidade de morte. $\mathrm{O}$ conjunto dos estudos descritos a seguir ilustra o impacto da criança com câncer no sistema familiar.

Em estudo exploratório descritivo, foram desveladas as experiências e necessidades de membros de famílias rurais que possuíam uma criança com câncer, de modo a proporcionarem aos profissionais de saúde, ligados à oncologia pediátrica, maior conhecimento dos desafios adicionais enfrentados por estas famílias ${ }^{(1)}$. Vinte e cinco membros de dez famílias foram entrevistados e os dados coletados através de observação participante, chamadas telefônicas e diário de campo permitiram aos autores identificar as várias perspectivas dos membros familiares, integrá-las, para compor uma visão da família como um todo, demonstrar as similaridades e diferenças entre seus membros e fazer comparações entre as famílias que compuseram o estudo. A análise dos dados revelou oito temas: "a história do nosso diagnóstico; tentando destruir o inimigo; longe de nossa casa; vivendo a uma distância da nossa esperança; a vida é diferente, mas nós precisamos ir adiante; o suporte dos outros; toda nossa família é afetada".

Excluindo-se a particularidade destas famílias de viverem em uma área geográfica rural e, como conseqüência, revelarem necessidades específicas ligadas a esta condição, percebemos, pelos temas, que a experiência de ter uma criança com câncer na família desvela necessidades relacionadas às diversas fases ao longo do processo da doença. Na maioria das famílias, estas fases referem-se ao período pré-diagnóstico, ao diagnóstico propriamente dito, ao momento de reorganização e reestruturação familiar, à exacerbação de sintomas da doença, aos períodos de hospitalização, à necessidade de aprender habilidades para lidar com a doença e à tomada de decisão frente a qualquer mudança no percurso da doença.

A presença de uma criança com câncer afeta toda a família e tem, ainda, o potencial de romper profundamente a sua estrutura, porém, à medida que seus membros adaptam-se à doença, seus papéis e responsabilidades podem mudar ${ }^{(2)}$. Os vários estados de desequilíbrio vivenciados ao longo 
da doença são substituídos por um estado de equilíbrio, quando todos os membros da família dominam suas necessidades emocionais e físicas ${ }^{(3)}$. Ao contrário, quando estas necessidades não são atingidas, permanece o estado de desequilíbrio e os indivíduos devem trabalhar continuamente para o restabelecimento do equilíbrio familiar.

Com o objetivo de obter maior entendimento sobre a experiência de pais chineses que tinham crianças diagnosticadas com leucemia linfocítica aguda (LLA), nove mães e oito pais de nove crianças foram entrevistadas, por duas vezes ${ }^{(4)}$. As entrevistas foram realizadas separadamente, a fim de se obter e comparar as visões individuais das mães e dos pais. O período de realização das entrevistas foi programado de forma a dar oportunidade aos pais de vivenciarem o dia-a-dia com a criança com leucemia, agendando a primeira entrevista, no mínimo, quatro semanas após a confirmação da doença, e a segunda, quatro meses após o diagnóstico. Esta última, geralmente coincidia com o término da terapêutica de indução e consolidação o que, muitas vezes, requeria freqüentes hospitalizações. Identificou-se quatro áreas relacionadas às experiências dos pais: 1) reações iniciais dos pais à confirmação diagnóstica da LLA; 2) método utilizado pelos pais para revelar o diagnóstico da criança para a própria criança e para os outros; 3) principais fontes de suporte social para os pais, e 4) mecanismos de enfrentamento da doença.

As experiências de pais chineses de crianças com LLA são similares à vivência de pais de outros países $^{(4)}$. A diferença encontrada refere-se aos recursos culturais, como diferentes religiões e crenças populares que fazem parte das estratégias de enfrentamento. A esse respeito, a autora sugere a necessidade de futuros estudos sobre suporte religioso na cultura chinesa e aponta as seguintes implicações para a prática de enfermagem: a avaliação dos fatores familiares deve incluir o levantamento de recursos para enfrentamento da doença, fontes de estresse e a compreensão da experiência dos pais.

Com o objetivo de explorar também as experiências vivenciadas por membros de famílias cujas crianças tivessem leucemia linfocítica aguda (LLA) e, especificamente, o "tornar-se" paciente oncológico vivido pela família como um todo, foram entrevistados membros de quatro famílias e três profissionais de uma clínica pediátrica oncológica, em Virginia, $U S A^{(5)}$. Por ocasião do diagnóstico, as crianças tinham entre dois e seis anos de idade e encontravam-se em estágio de manutenção do tratamento há um ou dois anos, pelo menos, na época do estudo.

Dentre as quatro famílias estudadas, três contaram com os pais e as mães nas entrevistas e duas com a presença da criança afetada, no processo de coleta de dados, verbalizando suas impressões. Esta pesquisa é importante, pois se diferencia metodologicamente da maioria daquelas que tratam deste tema, por trazer para a entrevista pai, mãe e criança. A combinação dos depoimentos dos casais e suas crianças com câncer fortalecem os dados da pesquisa, uma vez que facilitou a compreensão das vivências de todos os membros, individualmente e da família como um todo. Em outros trabalhos, comumente apenas um dos membros da família é entrevistado e suas verbalizações são generalizadas para a família, embora não represente um conhecimento produzido no âmbito familiar ${ }^{(6)}$.

O "tornar-se" paciente oncológico foi definido como: tentando viver o mais normal possível; aceitando que a vida não é mais a mesma; entendendo o que você tem que fazer e aceitando o que você não pode mudar/vivendo com descontentamentos. Este processo foi mediado por (1) contextos familiares externos, que incluem relações matrimonias e redes de suporte; (2) contextos de experiências, que abrangem experiências negativas e positivas durante o tratamento e experiências anteriores de doenças, e (3) contextos internos, incluindo estratégias de sobrevivência pessoal, atitudes e perspectivas pessoais ${ }^{(5)}$. A compreensão desse processo desse ser considerada por aqueles que prestam cuidado às crianças com câncer e suas famílias.

Em estudo qualitativo realizado com quatro pais e doze mães de quinze crianças e adolescentes suecos com câncer foram identificadas as dificuldades dos pais e os seus efeitos sobre a vida dos mes$\operatorname{mos}^{(7)}$. Os problemas encontrados legitimam suas preocupações, que coincidem com as descritas em estudos anteriores ${ }^{(1-5,8)}$. Entretanto, este trabalho acrescenta uma importante contribuição - avaliando a qualidade do cuidado - que não havia sido previamente descrita.

A qualidade do cuidado foi avaliada pelos pais através do grau de confiança e simpatia transmitido pela equipe médica e de enfermagem; da experiência profissional, conhecimento e habilidades em lidar com crianças; do oferecimento de informações sobre a doença e terapêutica, principalmente no início do tratamento e da disponibilidade de equipamentos na unidade para a criança, como gravador e vídeo $^{(7)}$. Na avaliação dos aspectos do cuidado, os pais indicaram a necessidade de um relacionamento efetivo entre profissionais e familiares, conhecimento específico para a assistência à criança e sua família e uma estrutura física adequada às necessidades da criança/adolescente em seu processo de crescimento e desenvolvimento.

mento e desenvolvimento.
Crianças com câncer e suas famílias 
Lucila C. Nascimento

Semiramis M. M. Rocha

Virginia H. Hayes

Regina A. G. de Lima
Processo de adaptação e estratégias utilizadas pelos pais diante da doença:

Vinte e seis mães e seis pais fizeram parte de um estudo sobre o processo de adaptação de pais chineses ao câncer infantil ${ }^{(8)}$, sendo os dados coletados através de entrevistas individuais e em grupo. Os resultados sugerem que o processo de adaptação dos pais se dá de forma dinâmica, com a modificação de suas habilidades e estratégias de enfrenta-mento da doença, de acordo com o momento clínico pelo qual estão passando. Os autores identificaram cinco fases, que compõem este processo de adaptação: enfrentando o tratamento, mantendo a integridade familiar, estabelecendo suporte, mantendo o bem-estar emocional e buscando por significado espiritual. Outro aspecto que merece atenção neste estudo foram os sujeitos da pesquisa, representados por mães, na sua maioria, indicando serem estas as principais cuidadoras da criança doente. Porém, mães e pais frequientemente têm diferentes perspectivas sobre suas preocupações e, então, como um pequeno número de pais foi entrevistado, mais dados são necessários para caracterizar o processo de adaptação destes, diante do câncer de seus filhos.

Outro estudo quali-quantitativo desenvolvido na China investigou as reações fisio-psicológicas de pais chineses frente ao tratamento e morte da criança com câncer ${ }^{(9)}$. Oitenta e nove famílias, cujas crianças encontravam-se em diversas fases do tratamento ou que haviam morrido, participaram da pesquisa. Os pais foram investigados a respeito de resfriado, dor de cabeça, tontura, diminuição do apetite e perda de peso, tendo as mães apresentado maior número de reações que os pais. Pais e mães relataram diminuição de apetite, perda de peso e dificuldades para dormir, seguidos de dor de cabeça, tontura e resfriado. $\mathrm{O}$ estágio da doença influenciou o tipo de reação dos pais, sendo a morte da criança identificada como o fator que gerou maior estresse. A relação entre o estágio da doença e as reações dos pais foi sustentada em outros estu$\operatorname{dos}^{(7-8,10)}$. Entretanto, em investigação sobre os níveis de angústia de pais, aos seis meses e um ano após o diagnóstico do câncer, não foram encontradas diferenças significativas ${ }^{(11)}$. Os níveis de angústia mantiveram-se altos, independente da fase da doença, evidenciando a importância de prover suporte para os pais ao longo de todo o processo.

A revisão da literatura tem apontado necessidades específicas dos pais nas diferentes fases da doença. Retomar o equilíbrio familiar, procurando incorporar a criança à rotina doméstica e tentando adaptá-la ao novo estilo de vida, tem sido um objetivo dos pais. Paralelamente à necessidade de retomar a "normalidade", estes buscam substituir os sentimentos de "incerteza" que permeiam sua experiência, pela "certeza" de que a doença está sob controle ou que está sendo manejada adequadamente ${ }^{(12)}$. Receber informações sobre o tratamento/ estado da criança; realizar parcerias com os profissionais de saúde, participando do cuidado; formar grupos de suportes individuais e/ou grupais, entre pais com crianças com doenças similares e profissionais, têm sido outras necessidades apontadas $^{(12-13)}$.

A fase de cuidados paliativos foi investigada em um estudo qualitativo ${ }^{(13)}$, tendo como sujeitos oito mães e quatro pais de crianças falecidas com câncer. Os dados foram coletados entre um e três anos após o falecimento. Os temas identificados nos depoimentos foram: a necessidade de reconhecer a criança como especial, em virtude de suas condições, enquanto os pais se esforçavam para preservar aspectos de uma infância "normal"; a necessidade de sentirem-se conectados e cuidados pelos profissionais de saúde e a necessidade de conservar a responsabilidade do trabalho de ser pai e mãe da criança, apesar dela estar morrendo. As necessidades apresentadas evidenciam a participação dos pais no cuidado das crianças, ressaltando o desafio para a enfermagem na inclusão dos mesmos no planejamento e na intervenção de suas ações.

A literatura internacional apresenta um conceito para definir o enfrentamento à doença (coping) $\mathrm{e}$ este conceito foi trazido para a oncologia pediátrica, mais especificamente relacionado ao modo como os pais enfrentam o câncer infantil ${ }^{(2,14-17)}$. Enfrentamento tem sido definido como sendo as ações e pensamentos utilizados para lidar com os desgastes e estresses da doença e suas conseqüências ${ }^{(2)}$. Embora haja um aumento no conhecimento resultante dos estudos conduzidos nesta área, a avaliação baseada em evidência mostra que os resultados úteis para o enfrentamento da doença ainda são limitados ${ }^{(14)}$. Além disso, aspectos metodológicos dos estudos podem estar comprometidos: muitos instrumentos usualmente utilizados não foram desenvolvidos por pais com crianças nessa situação; o uso de técnica única de coleta de dados para a avaliação, como só a aplicação de questionários ou checklists $^{(14)}$; instrumentos desenvolvidos para avaliar o enfrentamento individual podem não ser válidos, sensíveis e confiáveis, quando utilizados para mensurar o enfrentamento sob a perspectiva familiar ${ }^{(2)}$.

Os mecanismos utilizados pelos pais para enfrentar a doença são comumente apresentados na literatura de forma associada aos fatores estressantes experienciados pelos mesmos, no decorrer 
da doença. Apesar destes fatores variarem ao longo do tempo, geralmente estão associados ao momento do diagnóstico, às principais etapas do desenvolvimento da criança, aos cuidados de saúde necessários à continuidade do tratamento da criança e às experiências de exacerbação dos sintomas da doença e hospitalizações ${ }^{(14)}$.

Com o objetivo de descrever as estratégias familiares em resposta ao câncer infantil, foi desenvolvido um estudo com trinta e dois membros (sete mães, sete pais, seis crianças com câncer e doze irmãos sadios) de sete famílias, que tinham uma criança recentemente diagnosticada com câncer ${ }^{(2)}$. As estratégias identificadas foram: manejando o fluxo de informações, reorganizando papéis, avaliando prioridades, dando significado à doença, mudando orientação futura e manejando o protocolo terapêutico.

As estratégias selecionadas podem orientar intervenções de enfermagem no âmbito familiar, pois os esforços da autora para capturar a compreensão de todos os membros familiares, com suas similaridades, diferenças e interfaces e a integração dos conceitos teóricos no nível individual e familiar trazem a experiência do convívio com o câncer infantil na perspectiva da família. $\mathrm{O}$ estudo apresenta aspectos metodológicos que fortalecem seus resultados, como a variedade dos sujeitos da pesquisa que permite incorporar as idéias de vários membros das famílias - e os três pontos escolhidos para a realização das entrevistas, coincidindo com momentos da trajetória da doença que poderiam retratar significativas mudanças familiares.

\section{Processo de perda e luto frente à morte da criança:}

As experiências do processo de perda e luto dos pais pela morte da criança com câncer, bem como o processo de morrer vivenciado pela criança, têm sido investigados em vários estudos ${ }^{(18-26)}$. Os relatos dos familiares, principalmente das mães, revelam que, mesmo decorrido longo período após a perda da criança, emoções e sentimentos da convivência com a doença ainda permaneciam vivos. Sentimentos de dor, tristeza, angústia, saudade, depressão e dificuldades em continuar vivendo, aos poucos iam dando lugar a uma estabilidade gradual, com a construção de uma história de vida e morte da criança, relacionada à própria história das mães. As lembranças de momentos difíceis e tristes são substituídas por memórias da vida da criança como um todo. As mães percebem que a criança passou a ocupar um lugar específico, que está tudo bem e se sentem capazes de manejar a situação ${ }^{(18-19)}$. As participantes destes estudos estavam sendo acompanhadas através de reuniões grupais e o suporte recebido nesses grupos parece ter contribuído para que elas transpusessem as fases desse processo com maior naturalidade.

\section{CONCLUSÃO}

A revisão bibliográfica mostrou que a análise dos dados empíricos reforça a teoria sobre fases e transformações, ou seja, os momentos de maior desequilíbrio no sistema familiar são observados em determinadas fases, que podem estar ligados às etapas da própria doença ou aos marcos do desenvolvimento infantil. Porém, as pequenas particularidades individuais, culturais e regionais devem, também, ser levadas em conta, para que a assistência de enfermagem não seja padronizada de forma estereotipada e para que essas fases não sejam trabalhadas pela enfermagem, durante o cuidado, como um processo técnico, sem especificidades e individualidades.

Durante a leitura dos artigos científicos, tomamos um cuidado especial em relação à análise dos aspectos metodológicos no desenvolvimento de pesquisas com famílias. Observamos a preocupação dos autores em mostrar a diversidade de opiniões dos membros familiares, fato identificado nas entrevistas individuais e grupais realizadas com pais, mães, com as próprias crianças doentes, com irmãos e avós; o esforço dos autores em identificar as similaridades e diferenças das verbalizações dos membros das famílias; a relação entre os dados coletados e a interpretação do autor em termos da extensão de conceitos no âmbito familiar, quando os dados refletiam apenas aspectos individuais de membros das famílias e a utilização de diferentes técnicas de coleta de dados, procurando apreender ao máximo as especificidades dos objetos em investigação. Na revisão da literatura aqui apresentada, apenas dois $\operatorname{artigos}^{(1-2)}$ tinham o propósito de desenvolver conhecimento relativo à família, sendo os dados coletados com todos seus membros. Os demais coletaram depoimentos apenas dos pais e, com mais freqüência, das mães.

A presença de uma criança com uma doença como o câncer, afeta os relacionamentos familiares de diversas formas. Afeta, também, dimensões externas à estrutura familiar da criança, exigindo reflexões e adaptações, tanto por parte da criança, quanto dos seus familiares. Os estudos demonstram que a enfermagem precisa desenvolver métodos de abordagem que apreendam as suas necessidades de assistência, particularizando o cuidado de acordo com a singularidade de cada caso e evitando estereótipos ou preconceitos, os quais referem-se tanto às incapacidades da criança, quanto às limitações dos
Crianças com câncer e suas famílias

\author{
.
}


Lucila C. Nascimento Semiramis M. M. Rocha Virginia H. Hayes Regina A. G. de Lima pais em encontrarem formas criativas e positivas para lidar com dificuldades no processo de crescimento e desenvolvimento da criança. Em outras palavras, não se deve subestimar a competência dos pais e familiares, nem deixá-los desamparados quando necessitam de suporte.
Adequar os aspectos metodológicos para que todas as vozes dos familiares sejam representadas, selecionar o melhor método de coleta de informações das famílias e construir um conhecimento específico que considere as necessidades das famílias são questões fundamentais, que devem ser consideradas no desenvolvimento de futuras investigações.

\section{REFERÊNCIAS}

(1) Scott-Findlay S, Chalmers K. Rural families perspectives on having a child with cancer. J Pediatr Oncol Nurs 2001; 18(5):205-16.

(2) Clarke-Steffen L. Reconstructing reality: family strategies for managing childhood cancer. J Pediatr Nurs 1997; 12(5):278-87.

(3) Meleski DD. Families with chronically ill children: a literature review examines approaches to helping them cope. Am J Nurs 2002; 102(5):47-54.

(4) Wills BSH. The experiences of Hong Kong Chinese parents of children with acute lymphocytic leukemia. J Pediatr Nurs 1999; 14(4):231-8.

(5) Tarr J, Pickler RH. Becoming a cancer patient: a study of families of children with acute lymphocytic leukemia. J Pediatr Oncol Nurs 1999; 16(1):44-50.

(6) Nascimento LC. Crianças com câncer: a vida das famílias em constante construção. [tese] Ribeirão Preto (SP): Escola de Enfermagem de Ribeirão Preto/USP; 2003

(7) Enskär K, Carlsson M, Golsäter M, Hamrin E, Kreuger A. Parental reports of changes and challenges that result from parenting a child with cancer. J Pediatr Oncol Nurs 1997; 14(3):156-63.

(8) Yeh CH, Lee TT, Chen ML. Adaptational process of parents of pediatric oncology patients. Pediatr Hematol Oncol 2000; 17(2):119-31.

(9) Martinson IM, Liu-Chiang CY, Yi-Hua L. Distress symptoms and support systems of Chinese parents of children with cancer. Cancer Nurs 1997; 20(2): 94-9.

(10) Allen R, Newman SP, Souhami RL. Anxiety and depression in adolescent cancer: findings in patients and parents at the time of diagnosis. European $\mathrm{J}$ Cancer 1997; 33(8):1250-5.

(11) Sloper P. Predictors of distress in parents of children with cancer: a prospective study. J Pediatr Psychol 2000; 25(2):79-91.

(12) Fisher HR. The needs on parents with chronically sick children: a literature review. J Adv Nurs 2001; 36(4):600-7.

(13) James L, Johnson B. The needs of parents of pediatric oncology patients during the palliative care phase. $\mathrm{J}$ Pediatr Oncol Nurs 1997; 14(2):83-95.
(14) Melnyk BM, Feinstein NF, Moldenhouer Z, Small L. Coping in parents of children who are chronically ill: strategies for assessment and intervention. Pediatr Nurs 2001; 27(6):548-58

(15) Costa Jr AL, Coutinho SMG, Kanitz S. O enfrentamento do câncer em crianças: a intervenção da psicologia. Pediatr Mod 2000; 36(5):330-3.

(16) Muscari M. Coping with chronic illness. Am J Nurs 1998; 98(9):20-2.

(17) Last BF, Grootenhuis MA. Emotions, coping and the need for support in families of children with cancer: a model for psychosocial care. Patient Educ Couns 1998; 33(2):169-79.

(18) Saiki-Craighill S. The grieving process of Japanese mothers who have lost a child to cancer, part I: adjusting to life after losing a child. J Pediatr Oncol Nurs 2001a; 18(6):260-7.

(19) Saiki-Craighill S. The grieving process of Japanese mothers who have lost a child to cancer, part II: establishing a new relationship from the memories. J Pediatr Oncol Nurs 2001b; 18(6):268-75.

(20) Curnick S, Harris A. The dying child. In: Langton H, editor. The child with cancer: family-centered care in practice. Edinburgh: Baillière Tindall; 2000. p. 355-85.

(21) Davies B, Deveau E, de Veber B, Howell D, Martinson I, Papadatou D et al. Experiences of mothers in five countries whose child died of cancer. Cancer Nurs 1998; 21(5):301-11.

(22) Durham E. Caring for Cody. Am J Nurs 1998; 98(4):42-4

(23) Collins JJ, Stevans MM, Cousens P. Home care for dying child: a parent's perception. Aust Fam Physician 1998; 27(7):610-4.

(24) Sirkiä K, Saarinen UM, Ahlgren B, Hovi L. Terminal care of the child with cancer at home. Acta Paediatr 1997; 86(10):1125-30.

(25) Papadatou D. Training health professionals in caring for dying children and grieving families. Death Stud 1997; 21(6):575-600.

(26) Nesbit MJ, Hill M, Peterson N. A comprehensive pediatric bereavement program: the patterns of your life. Crit Care Nurs Q 1997; 20(2):48-62. 